\section{Educação em saúde: ensinando sobre a leishmaniose tegumentar americana}

\author{
Health education: teaching about \\ American tegumentary leishmaniasis
}

Claudia Maria Antunes Uchôa 1 Cathia Maria Barrientos Serra ${ }^{2}$ Ciléia de Melo Magalhães 2 Roger Magno Macedo da Silva ${ }^{3}$ Letícia Pinto Figliuolo 2 Cristianni Antunes Leal 3 Maria de Fátima Madeira 3

\author{
${ }^{1}$ Departamento de \\ Microbiologia e Parasitologia, \\ Instituto Biomédico, \\ Universidade Federal \\ Fluminense, Niterói, Brasil. \\ 2 Departamento de Saúde \\ Coletiva, Veterinária \\ e Saúde Pública, Faculdade \\ de Veterinária, Universidade \\ Federal Fluminense, \\ Niterói, Brasil. \\ 3 Escola Nacional de Saúde \\ Pública, Fundação Oswaldo \\ Cruz, Rio de Janeiro, Brasil.

\section{Correspondência} \\ C. M. A. Uchôa \\ Departamento de \\ Microbiologia e Parasitologia, \\ Instituto Biomédico, \\ Universidade Federal \\ Fluminense. \\ Rua Professor Hernani \\ de Mello 101, 3o andar \\ Niterói, RJ 24210-130, Brasil. \\ uchoa@radnet.com.br \\ claudiauchoa@vm.uff.br
}

\begin{abstract}
Knowledge on the circulation of American tegumentary leishmaniasis (ATL) and perception by the local population are highly relevant for developing a control program, since they mobilize the community for effective health measures. This work aimed to orient primary school teachers and pupils (grades 1 through 4) in the public school system in Maricá, Rio de Janeiro State, Brazil, on ATL, using talks, posters, and leaflets with an interactive approach. Talks were given in seven municipal schools, and activities were held with 1,314 pupils and 56 teachers. Leaflets were distributed to the pupils with activities to help consolidate the content. Two months later one school was chosen for postevaluation. Of the 213 pupils who had attended the talk, 198 answered a questionnaire, of whom 178 (89.9\%) could remember the disease and 125 (63.1\%) provided correct answers on transmission. Thus, informed children and teachers can function as information disseminators in health within their community and are therefore capable of contributing to control of endemic diseases.
\end{abstract}

Cutaneous Leishamaniasis; Health Education; Broadsides
A leishmaniose tegumentar americana (LTA) é uma zoonose que representa um problema de Saúde Pública no Brasil. Segundo Desjeux 1, os principais fatores de risco relacionados à leishmaniose cutânea zoonótica, em diversas regiões do mundo, são a urbanização, o desmatamento, o estabelecimento de novos povoados, a domesticação do ciclo de transmissão e o desenvolvimento de agricultura com construção de represas para irrigação. Na Região Sudeste do Brasil, esta doença apresenta um caráter de transmissão peri-domiciliar, principalmente pela adaptação do inseto vetor aos ambientes naturais modificados, possibilitando, desta forma, o envolvimento de animais domésticos 2 .

O conhecimento da população sobre a doença, nas regiões de sua ocorrência, inúmeras vezes é restrito, levando ao retardo na procura do diagnóstico e do tratamento, sendo as populações rurais de área endêmica as mais carentes de informação ${ }^{3}$. Na província de San José, Costa Rica, Dobles-Ulloa \& Perriard 4 concluíram que existe um risco grande de transmissão da leishmaniose cutânea no espaço doméstico e peridoméstico com alta incidência em crianças e mulheres. Eles relataram ainda que a prevenção deve basear-se em medidas caseiras, sendo a mulher o elemento principal no controle primário. Nesta região, o comportamento da infecção é diferente de outras áreas, onde os homens são mais afetados por se dedi- 
carem a atividades agrárias e estarem mais expostos à infecção 5 .

Na maioria das áreas onde existe a leishmaniose, o conhecimento sobre a enfermidade restringe-se, muitas vezes, a pessoas que já tiveram a doença ou àquelas que já tiveram casos na família ou vizinhos, ocorrendo desinformação sobre a transmissão e o tratamento, o que dificulta o estabelecimento de estratégias de controle 3,5.

No Maranhão, Brasil, Moreira et al.6, numa área de LTA, verificaram, por meio de questionário, que, apesar da população conhecer razoavelmente a doença considerando aspectos epidemiológicos, poucos são aqueles que fazem uso de medidas preventivas, contribuindo para a manutenção da doença.

Em áreas como Chocó na Colômbia, onde a população apresenta alto risco de aquisição de leishmaniose e a doença apresenta alta prevalência, Isaza et al. 7 verificaram que $94 \%$ da população estudada (345 pessoas) reconheceram a leishmaniose tegumentar como uma doença de pele, podendo apresentar-se na forma de úlceras, nódulos e manchas. Com relação à transmissão, 35\% relacionaram a doença com a picada de um inseto.

No Município de Maricá, Rio de Janeiro, Brasil, os relatos de casos humanos de LTA datam do início do século 8 . Nos últimos anos, tem sido observado no município um aumento da população humana associado a uma ocupação desordenada, principalmente próximo a encostas, ou matas, acarretando desequilíbrios ambientais que favorecem a instalação do ciclo extraflorestal da LTA.

Uchôa et al. ${ }^{9}$ estudaram a circulação da LTA em Maricá e verificaram a ocorrência de doença ativa no homem e em cães nas localidades de Inoã e Itaipuaçu.

O conhecimento sobre a circulação da doença em determinadas áreas, assim como a percepção da mesma pela população local, é de grande valia para o estabelecimento de campanhas de controle, pois mobiliza a comunidade em ações sanitárias 7,10,11,12,13,14.

Baseado nestes dados, o presente trabalho teve como objetivo esclarecer alunos e professores do ensino fundamental de Escolas Municipais de Maricá, por meio de palestras, cartazes e cartilhas, visando à conscientização do grupo e à sensibilização da comunidade em relação à LTA.

\section{Materiais e métodos}

O grupo trabalhado constituiu-se por alunos do ensino fundamental (1 1 a a 4 a série) e profes- sores de sete Escolas Municipais dos distritos de Inoã (3o) e Itaipuaçu (4ㅇo), pertencentes ao Município de Maricá, mediante prévia autorização da Secretaria de Educação de Maricá. Foram ministradas palestras e atividades ao grupo, durante os anos letivos de 1999 e 2000.

Todo o material utilizado nas atividades foi confeccionado pelos autores. Aos alunos, foi ministrada uma palestra por professores universitários e pesquisadores, com a participação de bolsistas e estagiários, nas salas de aulas das escolas, com auxílio de cartazes, sendo mostrados o inseto vetor e as lesões no homem e em cães, bem como discutido o mecanismo de transmissão e as formas de controle, levando em conta a experiência individual (Figura 1).

Após a palestra, para fixação do assunto, foi entregue uma cartilha (Figuras 2 e 3) com informações sobre a doença, e duas atividades para serem realizadas pelos alunos sob a supervisão dos palestrantes. Foram levados exemplares do inseto vetor para que os alunos e professores pudessem reconhecê-lo. As atividades na escola tiveram a duração de três horas.

Aos professores das escolas foram ministradas palestras com diapositivos e conteúdo mais profundo, uma semana antes das atividades em sala de aula.

Cerca de dois meses após as atividades, escolheu-se aleatoriamente uma escola, na qual aplicou-se um questionário com perguntas fechadas aos alunos para avaliação do conhecimento adquirido.

\section{Resultados}

Nas sete Escolas Municipais, foram ministradas palestras e atividades a 1.314 alunos e 56 professores (Tabela 1).

Na escola eleita para avaliação (Escola E), dos 213 alunos que assistiram à palestra 198 responderam ao questionário. Destes, 178 (89,9\%) lembravam da doença, e $125(63,1 \%)$ responderam corretamente como ocorria a transmissão. Visando minimizar a interferência nas atividades das escolas e a um melhor aproveitamento do tempo nos dias de atividade, não foi possível determinar a freqüência individual dos alunos nem no dia da palestra nem no dia do questionário.

\section{Discussão}

A LTA em Maricá ocorre na forma de casos esporádicos, restritos a algumas áreas mais carentes e próximas a matas 9 , sendo que o co- 


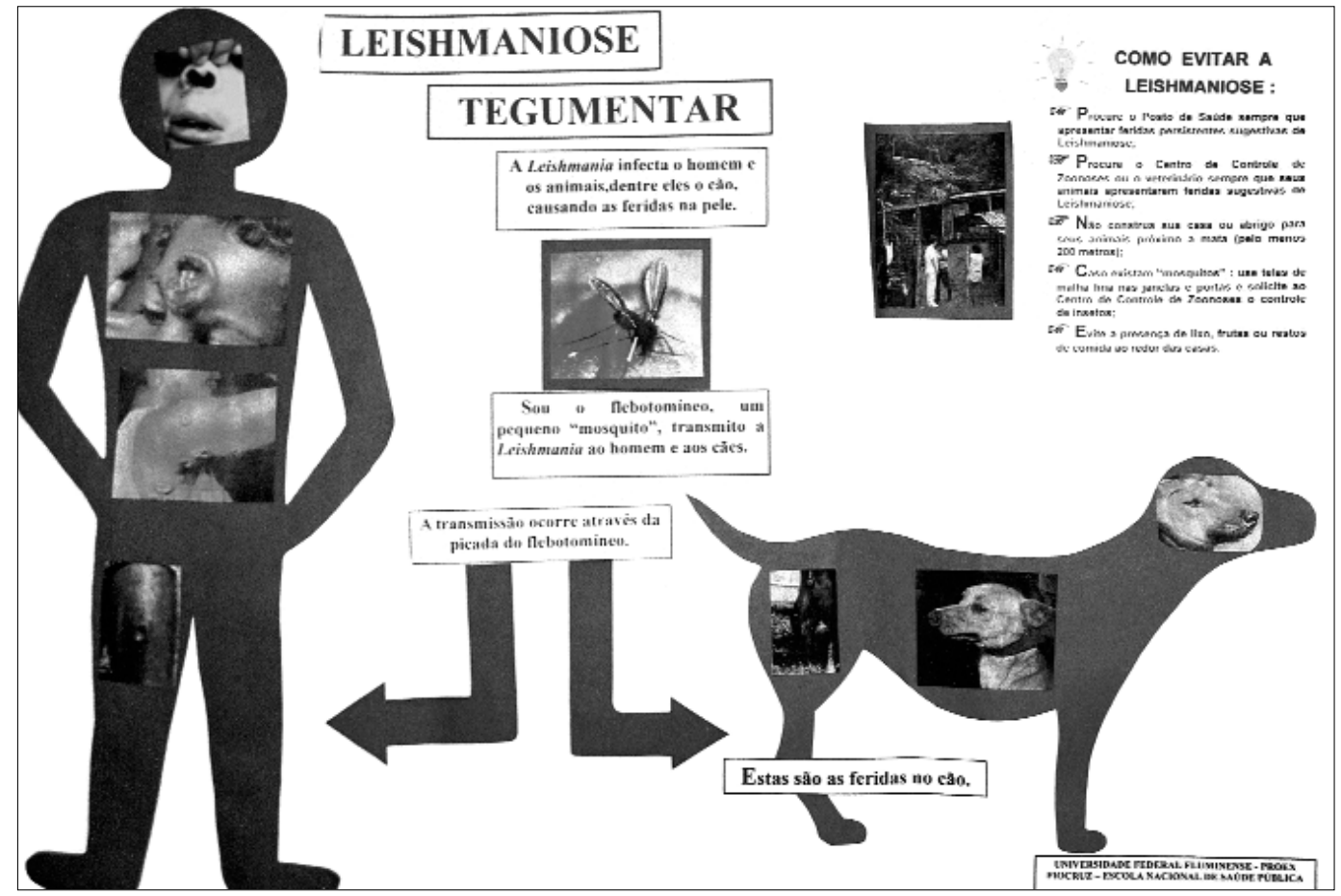

nhecimento sobre a mesma restringe-se a indivíduos que já vivenciaram a enfermidade ou tiveram pessoas de sua família ou vizinhos com a doença. Tal fato também foi observado por Weigel et al. 5 em uma zona endêmica do Equador e por Netto et al. 10 em Três Braços, na Bahia, Brasil. Em relação à leishmaniose visceral, no Maranhão, Gama et al. 3 observaram que a população local tinha noções básicas sobre a enfermidade, porém pouco sabiam sobre a prevenção e terapêutica.

Neste trabalho, durante as palestras, poucos professores ou alunos relataram conhecer a doença, demonstrando apenas informação sobre doenças como dengue e malária. Na Escola D, alguns alunos relataram conhecer a leishmaniose, pois uma das alunas apresentava cicatriz no rosto, tendo relatado ter adquirido a doença quando tinha cerca de um ano. Caldas et al. 15 observaram que os fatores de risco associados à leishmaniose visceral em crianças, no Nordeste do Brasil, variavam de acordo com padrões da população, sendo o risco maior em crianças com idades iguais ou superiores a 2 anos, estando associado com localização da residências e a casos em familiares.
Isaza et al. 7 verificaram que, numa área endêmica da Colômbia, 94\% dos habitantes conheciam a leishmaniose como uma doença de pele, e 35\% relacionavam a transmissão ao inseto. Neste estudo, após as palestras, pôde-se verificar que $89,9 \%$ das crianças lembravam do assunto, e $63,1 \%$ sabiam como ocorria a transmissão, demonstrando um excelente aprendizado em relação à leishmaniose, podendo atuar na formação da consciência sanitária em sua comunidade. Da mesma forma, Madeira et al. 14, estudando estratégias para controle da dengue, verificaram que alunos da $5 \underline{a}$ e $6 \underline{a}$ série, após intervenção didática, tornaram-se mais aptos em reconhecer o ciclo e a importância dos mosquitos para a saúde, bem como evidenciar as medidas de controle mais viáveis e eficientes. Também observaram duas vezes menos criadouros em suas residências do que na daqueles que não sofreram intervenção.

Pupulim et al. 12, estudando enteroparasitoses em escolares, verificaram o esclarecimento da população alvo após as atividades propostas, embora não pudessem medir a mudança de hábitos, uma vez que esta estaria vinculada a fatores ambientais e sociais associa- 


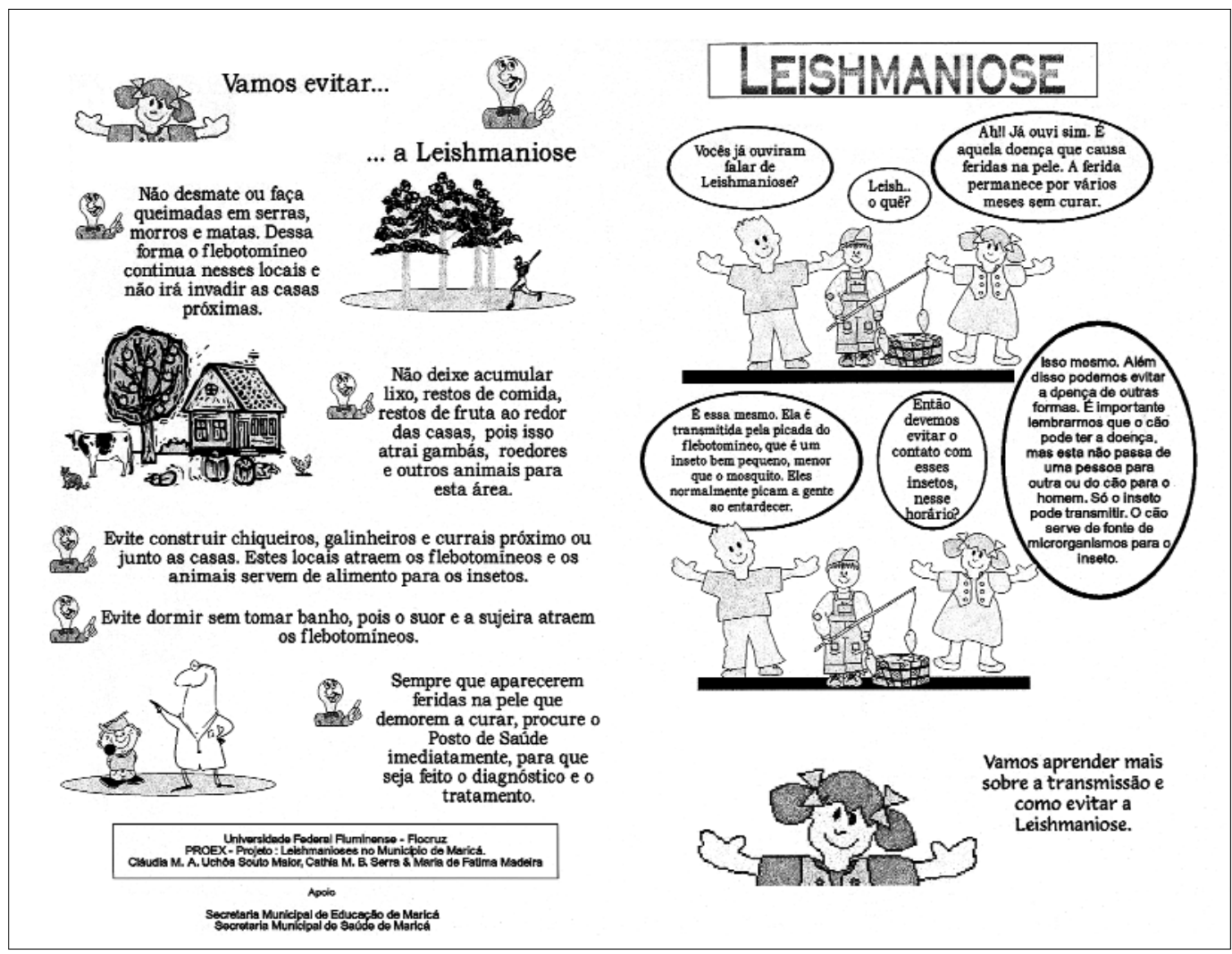

dos a condições subumanas e de miserabilidade, que, muitas vezes, impede a comunidade de mudar ou buscar alternativas para seus problemas.

Weigel et al. 5 relataram que a maioria das pessoas entrevistadas numa área endêmica no Equador conhecia a enfermidade, porém poucos sabiam que a transmissão ocorria por meio da picada do flebotomíneo. Segundo DoblesUlloa \& Perriard 4, na província de San José, Costa Rica, as mulheres são responsáveis pela manutenção da área que envolve a casa num raio de trinta metros e dos animais que vivem neste local (galinhas, cachorros, patos), porém elas não têm consciência dos animais envolvidos na transmissão, assim como a maioria considera o espaço doméstico como área que oferece maior segurança. Estes autores sugerem que o controle deveria fornecer informações amplas sobre a doença às mulheres visando ao aumento da segurança no espaço doméstico.

Santos et al. 13 também propõem a participação comunitária no controle de grandes en- demias, uma vez que o controle da LTA, direcionado ao vetor e aos reservatórios, tem esbarrado em dificuldades operacionais, representadas pelas condições ambientais em que a zoonose prevalece.

Moreira et al. 6, estudando uma área de LTA no Maranhão, concluíram que existe a necessidade da implantação de grupos de notificação e equipes de educação em saúde para orientar a prevenção e esclarecer sobre as formas de transmissão.

Neste estudo, a informação foi apresentada ao grupo pontualmente por meio de atividades em um único dia, não tendo sido avaliado o conhecimento prévio. Pôde-se perceber a aquisição do conhecimento sobre a leishmaniose, bem como em relação à transmissão, pela maioria das crianças, embora restritos a uma pequena amostra. Tal fato demonstra a importância da educação em saúde com a comunidade, com referência as principais doenças circulantes, visando a uma melhoria na qualidade de vida da população. 


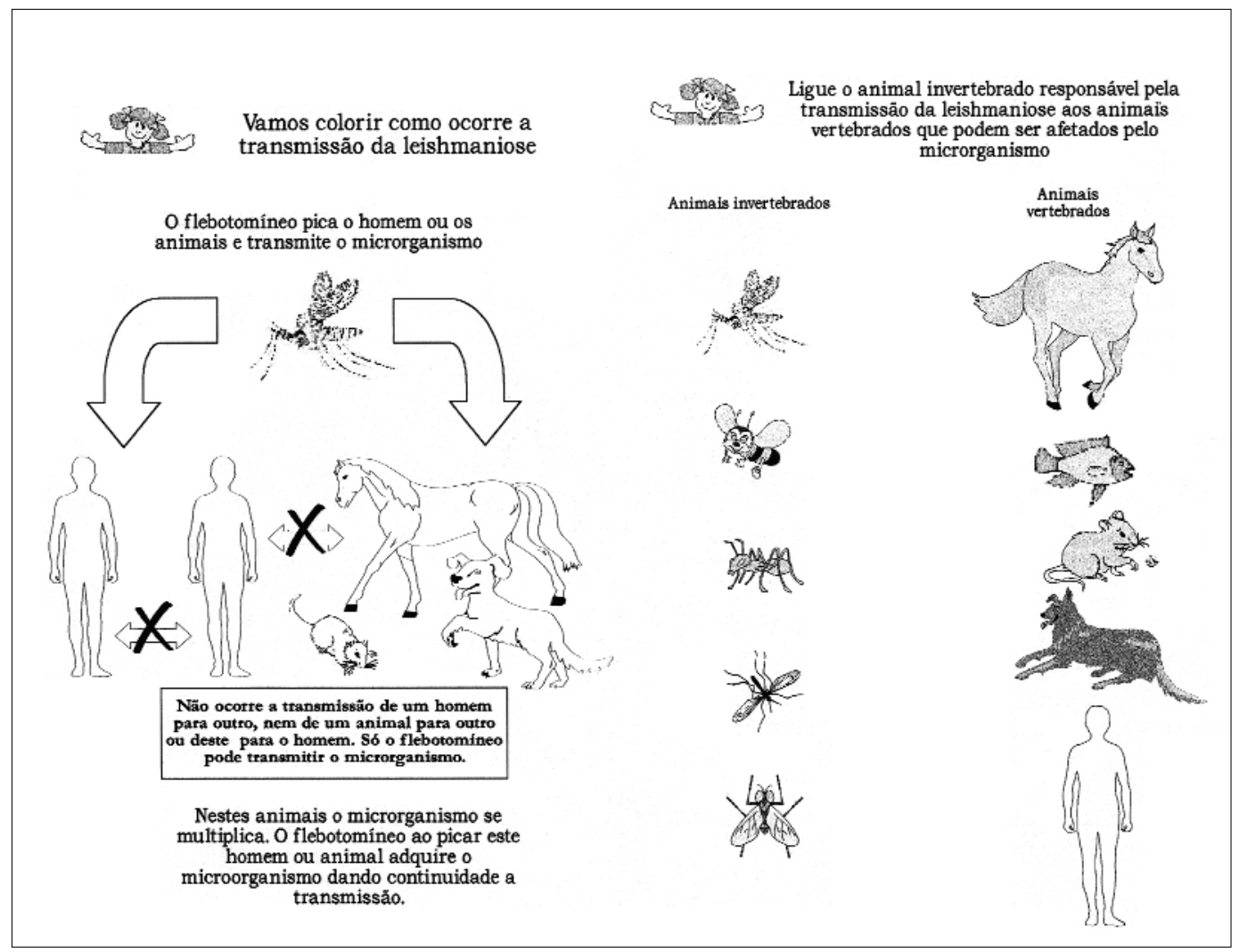

Pupulim et al. 12 relataram a importância de iniciar o processo de conscientização na população escolar, a qual deverá levar a informação a seus lares, podendo auxiliar na criação de uma rede básica de atenção primária e comunitária, assim como o proposto por Costa et al. 11. Desta forma, a criança poderá sair da letargia que caracteriza as comunidades desassistidas, tornando-se mais capaz de exercer sua cidadania 12.

Casavechia et al. 16 relatam que o acompanhamento de pacientes com LTA e as medidas educacionais são de fundamental importância para que o grupo social assuma um maior controle sobre sua saúde e vidas, sendo também o ponto de partida para a tomada de consciência e a incorporação de hábitos preventivos.

O esclarecimento da população torna-se imprescindível como ferramenta auxiliar no controle da LTA. Logo, crianças e professores informados podem funcionar como difusores do assunto em sua residência e comunidade, sendo capazes de atuar de forma relevante no controle de endemias.
Tabela 1

Número de alunos e professores das Escolas Municipais de Maricá, Rio de Janeiro, Brasil, do 3o e 4o Distrito (Inoã e Itaipuaçu), que participaram do trabalho de Educação sobre leishmaniose, nos anos de 1999 e 2000.

\begin{tabular}{llcc}
\hline Escola Municipal & Distrito & Número de alunos & $\begin{array}{c}\text { Número de } \\
\text { professores }\end{array}$ \\
\hline A & Itaipuaçu & 342 & 9 \\
B & Itaipuaçu & 220 & 6 \\
C & Itaipuaçu & 66 & 6 \\
D & Itaipuaçu & 150 & 6 \\
E & Inoã & 213 & 12 \\
F & Inoã & 285 & 14 \\
G & Inoã & 38 & 3 \\
Total & & 1.314 & 56 \\
\hline
\end{tabular}




\section{Resumo}

Os conhecimentos sobre a circulação da leishmaniose tegumentar americana (LTA), assim como sua percepção pela população local, são de grande valia para o estabelecimento de campanhas de controle, pois mobiliza a comunidade em ações sanitárias. Este trabalho teve como objetivo esclarecer alunos e professores do ensino fundamental (1 1 a a 4 a série) das Escolas Municipais de Maricá sobre a LTA, por meio de palestras, cartazes e cartilhas com atividades, visando à conscientização do grupo. Em sete Escolas Municipais, foram ministradas palestras e atividades a 1.314 alunos do ensino fundamental e 56 professores. Foram também distribuídas cartilhas aos alunos com atividades para a fixação do assunto. Após dois meses, uma escola foi eleita para avaliação (Escola E), sendo que, dos 213 alunos que assistiram a palestra, 198 responderam ao questionário, 178 (89,9\%) lembravam da doença, e $125(63,1 \%)$ responderam corretamente como ocorria a transmissão. Assim, crianças e professores informados podem funcionar como difusores da informação em Saúde na sua comunidade, sendo capazes de atuar de forma relevante no controle de endemias.

Leishmaniose Americana; Educação em Saúde; Impressos Avulsos

\section{Colaboradores}

C. Uchoa contribui na redação do manuscrito. Os demais autores contribuiram com opiniões, como na reorganização de resultados, inclusão de referências e análise dos resultados.

\section{Agradecimentos}

À Pró-Reitoria de Extensão (PROEX) da Universidade Federal Fluminense e à Escola Nacional de Saúde Pública da Fundação Oswaldo Cruz pelo apoio na realização dos trabalhos de campo e laboratorial. À Secretaria de Educação de Maricá, a todas as diretoras, professoras e alunos das Escolas Municipais de Maricá pela participação, oportunidade e compreensão. 


\section{Referências}

1. Desjeux P. The increase in risk factors for leishmaniasis worldwide. Trans R Soc Trop Med Hyg 2001; 95:239-43.

2. Marzochi MCA, Marzochi KBF. Tegumentary and visceral leishmaniasis in Brazil - emerging anthropozoonosis and possibilities for their control. Cad Saúde Pública 1994; 10:359-75.

3. Gama MEA, Barbosa JS, Pires B, Cunha AKB, Freitas AR, Ribeiro IR, et al. Avaliação do nível de conhecimento que populações residentes em áreas endêmicas têm sobre leishmaniose visceral, estado do Maranhão, Brasil. Cad Saúde Pública 1998; 14:381-90.

4. Dobles-Ulloa A, Perriard C. Representaciones, actitudes y practicas respecto a la leishmaniasis cutánea en la población del Cantón de Acosta, Provincia de san José, Costa Rioca. Estudio Antropológico exploratório. Cad Saúde Pública 1994; 10: 181-9.

5. Weigel MM, Armijos RX, Racines RJ, Zurita C, Izurieta R, Herrera E, et al. Cutaneous leishmaniasis in subtropical Ecuador: popular perceptions, knowledge, and treatment. Bull Pan Am Health Organ 1994; 28:142-55.

6. Moreira RCR, Rebelo JMM, Gama MEA, Costa JML. Nível de conhecimento sobre leishmaniose tegumentar americana (LTA) e uso de terapias alternativas por populações de uma área endêmica da Amazônia do Maranhão, Brazil. Cad Saúde Pública 2002; 18:187-95.

7. Isaza DM, Restrepo BN, Arboleda M, Casas E, Hinestroza H, Yurgaqui T. La leishmaniasis: conocimientos y praticas en poblaciones de la Costa del Pacífico de Colombia. Rev Panam Salud Publica 1999; 6:177-84.

8. Dutra-e-Silva O. Sobre a leishmaniose tegumentar e seu tratamento. Mem Inst Oswaldo Cruz 1915; 7:141-213.
9. Uchôa CMA, Serra CMB, Duarte R, Magalhães CM, Macedo RS, Theophilo F, et al. Aspectos sorológicos e epidemiológicos da Leishmaniose Tegumentar Americana canina em Maricá, Rio de Janeiro, Brasil. Rev Soc Bras Med Trop 2001; 34: 563-8.

10. Netto EM, Tada MS, Golightly L, Kalter DC, Iago E, Barreto AC, et al. Conceitos de uma população local a respeito da leishmaniose mucocutânea em uma área endêmica. Rev Soc Bras Med Trop $1985 ; 18: 33-7$

11. Costa JML, Vale KC, França F, Coatá MAF, Silva JO, Lago EL, et al. A leishmaniose tegumentar americana em uma região endêmica como fator de mobilização comunitária. Rev Soc Bras Med Trop 1994; 27:255-7.

12. Pupulim ART, Guilherme ALF, Falavigna DLM, Araújo SM, Fukushigue Y. Uma tentativa de orientar comunidades escolares no controle de enteroparasitoses. Rev Bras Anal Clin 1996; 28:130-3.

13. Santos JB, Lauand L, Souza GS, Macedo VO. Fatores sócio-econômicos e atitudes em relação à prevenção domiciliar da leishmaniose tegumentar americana, em uma área endêmica do Sul da Bahia, Brasil. Cad Saúde Pública 2000; 16:701-8.

14. Madeira NG, Macharelli CA, Pedras JF, Delfino MCN. Education in primary school as a strategy to control dengue. Rev Soc Bras Med Trop 2002; 35:221-6.

15. Caldas AJM, Costa JML, Silva AAM, Vinhas V, Barral A. Risk factors associated with asyntomatic infection by leishmania chagasi in north-east Brazil. Trans R Soc Trop Med Hyg 2002; 96:21-8.

16. Casavechia MTG, Araújo SM, Teixeira JJV, Lonardoni MVC. A leishmaniose tegumentar sob a perspectiva do paciente: resultado de uma prática educativa. Rev Bras Anal Clin 2002; 34:233-9.

Recebido em 28/Mar/2003

Versão final reapresentada em 21/Nov/2003

Aprovado em 27/Nov/2003 